\title{
artigo
}

Vasconcelos, L.; Farias, F.O.; Maia, J.M.A.; Carvalho, M.

Terapia Nutricional em um Hospital Pediátrico: Indicadores de Qualidade

\section{Terapia Nutricional em um Hospital Pediátrico: Indicadores de Qualidade}

\author{
Nutritional Therapy in a Pediatric Hospital: Quality Indicators \\ Terapia nutricional en un hospital pediátrico: indicadores de calidad
}

\begin{abstract}
RESUMO
Objetivo: Avaliar a qualidade da Terapia Nutricional Enteral em unidades de terapia intensiva pediátrica, através da aplicação de indicadores de qualidade. Métodos: Trata-se de um estudo de corte transversal prospectivo, composto por amostra de conveniência com pacientes de ambos os sexos, com idade de 0 até 18 anos incompletos, internados em unidades de terapia intensiva pediátrica e em uso exclusivo de terapia nutricional enteral. Foram aplicados três indicadores de qualidade e realizada a avaliação descritiva dos dados. Resultados: Participaram do estudo 20 pacientes, observou-se que $25 \%$ apresentaram pelo menos um episódio de diarreia, 45\% estiveram em jejum digestório por mais de 24 horas e não houve nenhum registro de obstrução de sonda. Conclusões: Dos resultados encontrados, apenas o indicador obstrução de sonda está dentro da meta proposta. Esse resultado corrobora com outros estudos realizados com adultos, porém são necessários estudos na área pediátrica para confrontar com os dados encontrados.
\end{abstract}

DESCRITORES: Indicadores de Qualidade em Assistência à Saúde; Terapia Nutricional; Nutrição Enteral.

\section{ABSTRACT}

Objective: To evaluate the quality of Enteral Nutritional Therapy in pediatric intensive care units, through the application of quality indicators. Methods: This is a prospective cross-sectional study, composed of a convenience sample with patients of both sexes, aged 0 to 18 years incomplete, hospitalized in pediatric intensive care units and in exclusive use of enteral nutritional therapy. Three quality indicators were applied and descriptive evaluation of the data was performed. Results: Twenty patients participated in the study, and it was observed that $25 \%$ had at least one episode of diarrhea, $45 \%$ were on digestive fasting for more than 24 hours and there was no record of tube obstruction. Conclusions: Of the results found, only the probe obstruction indicator is within the proposed target. This result corroborates with other studies conducted with adults, but studies in the pediatric area are needed to compare with the data found.

DESCRIPTORS: Quality Indicators in Health Care;Nutritional Therapy;Enteral Nutrition.

\section{RESUMEN}

Objetivo: Evaluar la calidad de la Terapia Nutricional Enteral en las unidades de cuidados intensivos pediátricos, mediante la aplicación de indicadores de calidad. Métodos: Se trata de un estudio prospectivo transversal, constituido por una muestra de conveniencia con pacientes de ambos sexos, de 0 a 17 años 11 meses y 29 días hospitalizados en unidades de cuidados intensivos pediátricos y en uso exclusivo de terapia. nutrición enteral. Se aplicaron tres indicadores de calidad y se realizó una evaluación descriptiva de los datos. Resultados: Tras aplicar los criterios de inclusión y exclusión, 20 pacientes participaron del estudio. Se observó que el $25 \%$ tuvo al menos un episodio de diarrea, el $45 \%$ había estado en ayuno digestivo por más de 24 horas y no hubo registro de obstrucción de la sonda en el estudio. Conclusiones: De los resultados encontrados, solo el indicador de obstrucción de la sonda se encuentra dentro del objetivo propuesto. Este resultado se corrobora con otros estudios realizados con adultos, pero son necesarios estudios en el área pediátrica, ya que esto permitirá comparar los datos encontrados con más deferencia. DESCRIPTORES: Indicadores de calidad en el cuidado de la salud; Terapia Nutricional; Nutrición Enteral.

RECEBIDO EM: 15/12/2020 APROVADO EM: 07/01/2021

\section{Larissa Vasconcelos}

Graduada em Nutrição pela Universidade Federal da Bahia (2014). Especialista em Saúde da Criança e Adolescente através da Residência Multiprofissional do Ministério da Saúde (2017).

ORCID: 0000-0003-1198-4204 


\section{Fernanda Orrico Farias}

Nutricionista graduada pela Universidade Federal da Bahia (2008). Especialista em Nutrição Clínica, com ênfase em Nutrição Pediátrica, pelo Programa de Residência em Nutrição Clínica da Escola de Nutrição da Universidade Federal da Bahia em parceria com a Secretaria Estadual de Saúde do Estado da Bahia (2011).

ORCID: 0000-0002-9746-6522

\section{Júlia Martins Azevedo Maia}

Possui graduação em Enfermagem pela Escola Bahiana de Medicina e Saúde Pública (2014). Especialista em Pediatria e Neonatologia pela Escola Bahiana de Medicina e Saúde Pública (2014-2015).

ORCID: 0000-0002-4711-2531

\section{Mirela Carvalho}

Bela em Nutrição pela Universidade Federal da Bahia (2003). Mestre em Desenvolvimento Regional e Urbano - Universidade Salvador - Unifacs. Especialista em Gestão em Saúde Pública.

ORCID: 0000-0003-1515-8540

\section{INTRODUÇÃO}

A o longo das últimas décadas, a Terapia Nutricional (TN) tem mostrado sua importância, principalmente por contribuir de maneira positiva nos desfechos clínicos de pacientes em estados críticos. A Terapia Nutricional Enteral (TNE) é parte importante durante o tratamento de crianças internadas em unidades de terapia intensiva, porém, apesar de sua eficiência a mesma não é isenta de efeitos adversos. ${ }^{1}$

Os Indicadores de Qualidade em Terapia Nutricional (IQTN) surgem como uma importante ferramenta para combater os eventos adversos que podem ter como consequências: o aumento da desnutrição hospitalar, a morbidade e mortalidade e a redução do custo benefício da TN. ${ }^{2}$ Os IQTN constituem um método quantitativo de avaliação da assistência nutricional num serviço de saúde. ${ }^{3}$

O objetivo principal dos IQTN é garantir a segurança do paciente. Segurança na assistência à saúde significa evitar, prevenir e aprimorar resultados adversos e danos gerados pelo processo de assistência à saúde. A segurança do paciente inclui a redução e a mitigação de atos não seguros dentro do sistema de assistência à saúde, assim como a utilização de boas práticas para alcançar resultados ótimos para o paciente. ${ }^{4}$

A Força Tarefa de Nutrição Clínica do International Life Sciences Institute - Brasil (ILSI), visando o controle de qualidade em TN, publicou 36 indicadores que permitem avaliar na prática a qualidade com que a TN, seja enteral ou parenteral, está sendo conduzida. ${ }^{5}$ Posteriormente foi realizado um estudo para identificar os 10 IQTN considerados mais úteis, práticos, de fácil execução (simplicidade) e baixo custo. ${ }^{3} \mathrm{Na}$ escolha dos indicadores é preciso considerar que serão escolhidos para implementação, demandam tempo para a monitorização, a coleta criteriosa dos dados e, posteriormente a análise crítica desses dados, com identificação de possibilidades de melhoria. ${ }^{4}$

A segurança do paciente é uma questão que vem sendo discutida de forma global. As instituições hospitalares reconhecem a importância de reduzir ao mínimo aceitável os riscos de danos desnecessários associados à assistência em saúde para obtenção do certificado de qualidade. ${ }^{6}$ Todavia quando se trata de pacientes pediátricos, o item segurança precisa ser reforçado. No Brasil, os estudos relacionados à segurança do paciente são recentes e realizados em grande parte abordando a temática dos incidentes com medicamentos. ${ }^{7}$ Os estudos científicos envolvendo avaliação de IQTN em pediatria são escassos, e existem poucos registros do impacto dessa avaliação na rotina das instituições, de forma que garanta ao paciente a qualidade na assistência. ${ }^{8}$

Dessa forma se faz necessário conhecer e avaliar os IQTN na área de pediatria, a fim de conhecer as não conformidades e assim adotar estratégias para minimizar eventos adversos que possam culminar em maiores riscos de morbimortalidade, maior tempo de internação e maiores custos.

\section{MÉTODOS}

Trata-se de um estudo de corte transversal prospectivo, composto por amostra de conveniência, realizado com pacientes acompanhados nas três unidades de terapia intensiva pediátricas de um hospital pediátrico em Salvador, após submissão e aprovação da Plataforma Brasil, parecer $n^{\circ}$ 2.121.360, e da Comissão Avaliadora de Ensino e Pesquisa da própria instituição (CAAE: 64718216.0.0000.5033).

A coleta de dados ocorreu de junho de 2017 a julho de 2017 por um período de 30 dias. Foram incluídos no estudo pacientes de ambos os sexos, com idade de 0 até 17 anos 11 meses e 29 dias, em terapia nutricional enteral por via gástrica ou enteral, com gastrostomia ou jejunostomia por pelo menos 72 horas e cujo responsável legal concordou em participar da pesquisa após assinatura do Termo de Consentimento Livre e Esclarecido (TCLE). O TCLE foi escrito de acordo com a resolução CNS 466/2012, direcionada as pesquisas envolvendo seres humanos. Também foi confeccionado para pesquisa o Termo de Assentimento, que não dispensa o TCLE, porém não houve aplicação. Foram ainda aplicados os seguintes critérios de exclusão: terapia mista, suplementação oral e/ou parenteral e admissões de outras unidades hospitalares já em uso de terapia nutricional. 


\section{artigo}

A coleta de dados foi iniciada no primeiro dia de introdução da nutrição enteral, e posteriormente o acompanhamento foi diário até o momento de interrupção da terapia, óbito, alta hospitalar ou finalização do estudo. A coleta de dados ocorreu durante 30 dias contínuos e foi realizada pela pesquisadora principal nos prontuários dos pacientes, nos registros $\mathrm{da}$ equipe de enfermagem e inicialmente com os próprios responsáveis, através de questionário de admissão aplicado.

Os indicadores de qualidade aplicados foram propostos pela Força Tarefa em Nutrição Clínica do Comitê de Nutrição do ILSI Brasil. Os indicadores escolhidos para serem avaliados, devido a sua facilidade, utilidade e objetividade foram: frequência de diarreia, frequência de obstrução de sonda de nutrição e frequência de jejum digestório por mais de $24 \mathrm{~h}$ em pacientes em TNE. Considerou-se como diarreia ocorrência de 3 ou mais dejeções líquidas ou semilíquidas no período de $24 \mathrm{~h} .{ }^{9}$

Foi realizada análise descritiva dos dados, apresentando-os em frequências absolutas e relativas, com as médias e desvio padrão. O programa estatístico utilizado foi Statistical Package for Social Sciences (SPSS), versão 12.0.

Importante citar que a pesquisa respeitou as normas estabelecidas no Estatuto da Criança e do Adolescente (ECA).

\section{RESULTADOS}

Vinte pacientes atenderam aos critérios de elegibilidade da pesquisa e três foram excluídos. A média etária encontrada foi 256,2 dias, com variação entre 2 dias e 1460 dias, sendo $55 \%$ dos participantes do sexo masculino. Em relação a via de administração mais utilizada a sonda nasoenteral apresentou maior prevalência (55\%), seguindo da sonda orogástrica (40\%). A duração de tempo de permanência em uso de TNE exclusiva variou de 3 a 19 dias com a média de 8,1 \pm 4,33 dias. Entre as principais causas de internamento nas unidades de terapia intensiva o pós-operatório de cirurgias cardíacas tiveram maior ocorrência (35\%), seguindo de complica- ções respiratórias (30\%). A caracterização da população e as outras variáveis foram apresentadas na tabela 1 .

Os resultados da aplicação dos indicadores estão descritos na tabela 2. Dos resultados encontrados, apenas o indicador obstrução de sonda está dentro da meta proposta.

\section{DISCUSSÃO}

A prevalência de desnutrição entre pacientes críticos, especialmente aqueles com internamento prolongado, tem se mantido constante nos últimos anos. A intensa resposta metabólica ao trauma e a falha no fornecimento de apoio nutri- cional ideal durante o internamento nas unidades de terapia intensiva (UTIs) são alguns dos principais fatores que contribuem para a desnutrição nesse público. Embora a terapia de suporte nutricional não possa reverter ou evitar esta resposta, se houver falhas no fornecimento de nutrientes durante este estágio, resultará em excessos ou deficiências de nutrientes e provável desnutrição, que afetará diretamente os resultados clínicos. ${ }^{10}$

O objetivo das terapias de suporte nutricional para crianças criticamente doentes é aumentar os benefícios a curto prazo da resposta ao estresse metabólico e minimizar a longo prazo consequências no seu desenvolvimento. Avaliação precisa das neces-

\section{Tabela 1: Caracterização da população e da Terapia Nutricional Enteral}

\section{Características}

Média em dias

Gênero

Masculino

Feminino

Diagnóstico

Sepse

Respiratório

Cirurgia Cardíaca

Neoplasia

Cirurgia do TGla

Vias de administração

SOG ${ }^{\mathrm{b}}$

$\mathrm{SNE}^{\mathrm{C}}$

$\mathrm{SNG}^{\mathrm{d}}$

Média de tempo em TNE exclusiva (dias)

$\mathrm{a}=$ Trato Gastrointestinal; $\mathrm{b}=$ sonda orogástrica; $\mathrm{c}=$ sonda nasoenteral; $\mathrm{d}=$ sonda nasogástrica; TNE= Terapia Nutricional Enteral

Tabela 2: Resultado da aplicação dos indicadores de qualidade aplicados nos pacientes em uso de terapia nutricional enteral

Indicadores de Qualidade em Terapia Nutricional

Frequência de diarreia em pacientes com TNE

Frequência de obstrução de sonda de nutrição em pacientes em TNE

Frequência de jejum digestório por mais de $24 \mathrm{~h}$ em pacientes em TNE

$\mathrm{a}=$ Terapia Nutricional Enteral
$\mathrm{N}=20$

$256,2 \pm 405,2$

11

9

2

6

7

2

3

8

11

1

$8,1 \pm 4,33$ 
sidades energéticas e provisão de suporte nutricional ideal é importante objetivo do cuidado crítico pediátrico. A entrega desses nutrientes requer uma seleção cuidadosa do modo apropriado de alimentação e monitoração constante para garantir o sucesso da estratégia de alimentação. ${ }^{11,12}$

A aplicação de indicadores de qualidade em TNE é uma forma de promover a qualidade na assistência nutricional, pois os indicadores expressam em números a atenção nutricional e a atuação da Equipe Multidisciplinar em Terapia Nutricional (EMTN). Os passos a serem seguidos seriam a identificação do problema, o estabelecimento do padrão de referência a ser considerado, a análise dos dados quantitativos e a identificação de aspectos a serem controlados para alcance do padrão estabelecido. ${ }^{13}$ Assim, cumprir a meta dos indicadores de qualidade em TNE está relacionado à melhor recuperação nutricional e clínica dos pacientes.

No presente estudo, o indicador frequência de episódios de diarreia (25\%) foi superior à meta proposta $(<10 \%)$, porém inferior aos valores encontrados por Brandão e Rosa $(54,5 \%)$ em estudo com pacientes adultos com quadro crítico e em uso de terapia nutricional enteral. ${ }^{14} \mathrm{Tal}$ indicador tem como objetivo mensurar a ocorrência de diarreia naqueles pacientes que recebem nutrição enteral com o propósito de permitir a tomada de medidas preventivas e terapêuticas visando reduzir essa complicação. ${ }^{5}$

Em estudo conduzido por Cartolano et al. ${ }^{15}$ por meio da utilização de indicador de qualidade, a frequência de episódios de diarreia foi de $6,76 \%$, portanto, inferior a $10 \%$, conforme meta estabelecida. Já no estudo de Aranjues et al. ${ }^{13}$, no ano de 2005, 36\% dos pacientes apresentaram pelo menos 1 episódio de diarreia. Posteriormente, em 2006, esse número caiu para $17 \%$ dos pacientes. A monitorização da terapia através dos indicadores, e a intervenção da equipe multiprofissional possibilitou a diminuição do índice de diarreia no referido estudo.

O surgimento de diarreia durante a internação hospitalar é evento comum no doente crítico, com incidência de $2 \%$ a $92 \%$. Algumas das causas de diarreia na UTI seriam as medicações prescritas, uso de nutrição artificial, presença de infecções, presença de isquemia ou fístula intestinal, septicemia, hipoalbuminemia, dentre outras. Assim, a presença de diarreia em pacientes críticos recebendo nutrição enteral necessita ser criteriosamente investigada. ${ }^{14,16}$

\section{Neste estudo}

percebemos que

a ocorrência de

diarreia é uma

complicação na

UTI que não tem

sido mais causa

de interrupção

na administração

da TNE.

Neste estudo percebemos que a ocorrência de diarreia é uma complicação na UTI que não tem sido mais causa de interrupção na administração da TNE. Acredita-se que a grande variabilidade de fórmulas e suas especificidades colabora para não interrupção do tratamento dietoterápico.

Além disso, é importante estabelecer e padronizar a definição de diarreia, preferencialmente por meio de protocolo conduzido pela equipe multiprofissional, visando diagnosticar e tratar a diarreia associada à TNE no paciente grave. ${ }^{17}$
Em relação a avaliação da prevalência de obstrução de sonda de nutrição, o objetivo estratégico do indicador é mensurar o número de obstruções de sondas enterais, assim como o número de sondas retiradas devido a obstruções, naqueles pacientes em terapia nutricional enteral. Além disso, busca identificar as possíveis causas de obstrução permitindo a tomada de medidas preventivas e/ou corretivas. O ideal é que seu resultado seja inferior a $10 \%{ }^{5} \mathrm{Nes}-$ te estudo não se observou ocorrência de obstrução da sonda enteral, satisfazendo plenamente a meta proposta.

A administração da nutrição enteral é dificultada por fatores diretamente relacionados à terapia intensiva, porém, os problemas mecânicos com a sonda nasoenteral, como a obstrução da sonda, podem acarretar em prejuízos ao suporte nutricional. ${ }^{8}$

No presente estudo, $45 \%$ dos pacientes foram submetidos a jejum por períodos superiores a 24 horas, valor este muito superior à meta estipulada $(<10 \%)$. O objetivo deste indicador é verificar a ocorrência de interrupções da nutrição enteral por períodos superiores a 24 horas. O conhecimento deste indicador permite a adoção de medidas corretivas para minimizar o risco de desnutrição ou a piora do estado nutricional. $\mathrm{O}$ ideal é que um número menor ou igual a $10 \%$ dos pacientes seja submetido a jejum superior a 24 horas. ${ }^{5}$

A preparação para cirurgias, procedimentos e exames parecem ser interrupções necessárias e, segundo O'Meara e colaboradores $^{18}$, aproximadamente 9 horas são gastas com o jejum antes da cirurgia e 6,6 horas para procedimentos radiológicos sem adultos. É preocupante que alguns procedimentos podem não exigir jejum, ou o seu período pode ser encurtado. A adoção de protocolos de terapia nutricional incluindo protocolos de jejum, podem minimizar esse jejum prolongado.

Em outra pesquisa, após aplicação de indicadores de qualidade da terapia nutricional, Brandão e Rosa ${ }^{9}$ também não atenderam à meta preconizada visto que o número de pacientes adulto sem jejum superior a 24 horas $(18,2 \%)$ 


\section{artigo}

foi superior àquele desejável, porém, ainda inferior ao valor encontrado no presente trabalho.

Segundo recomendações da ESPEN os pacientes devem ser alimentados porque o jejum em UTIS está associado ao aumento da morbidade e mortalidade. ${ }^{19}$

Neste contexto, Heyland e colaboradores 20 discutem a tendência dos pacientes hospitalizados permanecerem em jejum ou receberem uma terapia nutricional inadequada com calorias insuficientes. Os autores afirmam que protocolos seriam úteis no sentido de diminuir o número e a duração dos períodos de je- jum ou interrupções da nutrição enteral, acelerando o início precoce da NE, aumentando o volume e reduzindo os obstáculos ao seu fornecimento.

\section{CONCLUSÃO}

O estudo possui algumas limitações entre elas a ausência de outros estudos na área de pediatria sobre indicadores de qualidade, importante para uma discussão mais específica. A falta de pesquisa sistemática e ensaios clínicos em vários aspectos de suporte nutricional em pacientes críticos pediátricos são impressionantes e existe uma necessidade de conceber ensaios multicêntricos nesta área de prática clínica. A extrapolação de dados da literatura de cuidados intensivos para adultos não é desejável e muitas das intervenções propostas em adultos terão que passar por análises criteriosas para serem aplicadas em crianças criticamente doentes. A outra limitação diz respeito ao tamanho amostral, no entanto os resultados aqui apresentados são muito similares aos descritos na literatura. Por fim, é necessária a constante avaliação da qualidade da terapia nutricional, como forma de monitorar essa parte importante da assistência à saúde. ๓

\section{REFERÊNCIAS}

1. Luz ERL, Mezzomo TR. Estado nutricional e indicadores de qualidade em terapia nutricional enteral em pacientes institucionalizados com paralisia cerebral. Demetra, 2015; 10(1),189-202.

2. Schneider APGC; Mendonça SS. Indicadores de Qualidade em Terapia Nutricional em Terapia Intensiva. Com. CiênciasSaúde. 2015; 26(3/4): 127-132

3. Waitzberg DL, coordenador. Indicadores de Qualidade em Terapia Nutricional: Aplicação e Resultados. São Paulo: ILSI Brasil,2010

4. Waitzberg DL, Enck CR, Miyahira NS, Mourão JRP, Faim MMR, Oliseski et all. ProjetoDiretrizes, TerapiaNutricional: Indicadores de Qualidade. Associação Médica Brasileira e Conselho Federal de Medicina, 2011.

5. Waitzberg DL, coordenador. Indicadores de Qualidade em Terapia Nutricional. São Paulo: ILSI Brasil,2008

6. Verotti CCG, Torrinhas RSMM, Cecconello I, Waitzberg DL. Selection of Top 10 Quality Indicators for Nutrition Therapy. Nutrition in Clinical Practice Volume 27 Number 2 April 2012 261-267

7. Ceniccola GD, Araújo WMC, Akutsu R. Development of a tool for quality control audits in hospital enteral nutrition. Nutr Hosp. 2014; 29(1): 102-120.

8. Nilesh MM, Charlene C and A.S.P.E.N. Board of Directors. A.S.P.E.N. Clinical Guidelines: Nutrition Support of the Critically III Child. Journal of Parenteral and Enteral Nutrition / Vol. 33, No. 3, May/June 2009

9. WHO,2013.Disponível em: http://www.who.int/mediacentre/ factsheets/fs330/en/index.html

10. Nilesh MM, Charlene C and A.S.P.E.N. Board ofDirectors. A.S.P.E.N. ClinicalGuidelines: NutritionSupportoftheCriticallylllChild. Journal of Parenteral and Enteral Nutrition / Vol. 33, No. 3, May/June 2009

11. Silva FM, Bermudes ACG, Maneschy IR, et al . O impacto da introdução precoce de terapia nutricional enteral na redução da morbimortalidade na terapia intensiva pediátrica: uma revisão sistemática. Rev. Assoc. Med. Bras. 2013, São Paulo, v. 59, n. 6, p. 563-570

12. Fernandes VPI, Lima $A$, Euzébio Junior $A A$, Nogueira R. Nutrição enteral em pediatria. ResidPediatr. 2013;3(3):67-75

13. Aranjues AL, Caruso L, Teixeira ACC, Soriano FG. Monitoração da terapia nutricional enteral em UTI: indicador de qualidade? 0 Mundo da Saúde. 2008;32(1):16-23

14. Brandão VL, Rosa LPS. Nutrição enteral em pacientes internados em unidade de terapia intensiva: análise dos indicadores de qualidade. Brasília Med 2013;50(3):200-205

15. Cartolano FC, Caruso L, Soriano FG. Terapia nutricional enteral: aplicação de indicadores de qualidade. RevBras Ter Intensiva. 2009; 21(4):376-383.

16. Manzoli de Sá JS, Marshall NG. Indicadores de Qualidade em Terapia Nutricional como ferramenta para avaliação da assistência nutricional em pacientes hospitalizados. Com. Ciências Saúde. 2014; 25(2): 127-140

17. Caruso, L. Indicadores de qualidade. In: Caruso, L.; Sousa, A. B. (Org.). Manual da equipe multidisciplinar de terapia nutricional (EMTN) do Hospital Universitário da Universidade de São Paulo - HU/USP. São Paulo: Hospital Universitário da Universidade de São Paulo, 2014. p. 127-132

18. O'Meara D, Mireles-Cabodevila E, Frame F, Hummell AC, Hammel J, Dweik RA, et al. Evaluationof delivery of enteral nutrition in criticallyillpatientsreceivingmechanicalventilation. Am J CritCare. 2008; 17(1): 53-61.

19. Singer $P$, Berger MM, Van denBerghe $G$, Biolo $G$, Calder $P$, Forbes $A$, et al. ESPEN Guidelineson Parenteral Nutrition: IntensiveCare. ClinNut. 2009; 28(1): 387-400.

20. Heyland DK, Cahill NE, Dhaliwal R, Sun X, Day AG, McClave SA. Impactof Enteral FeedingProtocolson Enteral Nutrition Delivery: Resultsof a MulticenterObservationalStudy. JPEN J Parenter Enteral Nutr. 2010; 34(6): 675-684. 